\title{
Dinámica de olas del tsunami
}

Yuri N. Skiba

\section{Resumen}

Este artículo considera la generación de un tsunami tectónico y explica dos fenómenos importantes que ocurren cuando las olas se acercan a la costa. Uno de ellos es la compresión de las olas, un aumento en sus amplitudes y una disminución en su velocidad cuando un tsunami entra en aguas poco profundas cerca de la costa. Como resultado, la energía potencial de las olas y su poder destructivo aumentan. Otro fenómeno que se comenta en el artículo es el colapso de las crestas de las olas cuando llegan a la costa. Éste es un proceso físico complejo, característico de muchas olas del mar y del océano.

Palabras clave: tsunami, amplificación de las amplitudes de olas, colapso de las crestas de las olas.

\section{TSUNAMI WAVE DYNAMICS}

\begin{abstract}
This article considers the generation of a tectonic tsunami and explains two important phenomena that occur when waves approach shore. One of the phenomena is the compression of the waves, an increase in their amplitudes and a decrease in their speed when a tsunami enters shallow waters near the coast. As a result, the potential energy of tsunami waves and their destructive power increase. Another phenomenon that is discussed is the collapse of the wave crests when they reach the shore. This is a complex physical process that is characteristic of many sea and ocean waves.
\end{abstract}

Keywords: tsunami, amplification of wave amplitudes, collapse of wave crests.

Recepción: 09/12/2020. Aprobación: 30/04/2021. Dol: http://doi.org/10.22201/cuaieed.16076079e.2021.22.5.6 
Se doctoró en Matemáticas y Física en la uRss en 1979. Actualmente es investigador Titular C, profesor en la unam y jefe de modelación de procesos atmosféricos en el Centro de Ciencias de la Atmósfera. Es autor de los libros Mathematical Problems of Dynamics of Viscous Barotropic Fluid on a Rotating Sphere (URSS, 1989), Application of adjoint equations to problems of dispersion and control of pollutants (USA, 2015), Mathematical problems of the dynamics of incompressible fluid on a rotating sphere (Suiza, 2017), Introducción a los Métodos Numéricos (2001); Métodos y Esquemas Numéricos: Un Análisis Computacional (2005); Introducción a la Dinámica de Fluidos (2009); Introducción a los Métodos de Dispersión y Control de Contaminantes (2011); y Fundamentos de los métodos computacionales en álgebra lineal (2018). Es además autor de 140 artículos científicos, 27 capítulos en libros y 54 artículos en memorias, y pertenece al Sistema Nacional de Investigadores, nivel I. Sus trabajos teóricos se centran en la existencia, unicidad, estabilidad y comportamiento asintótico de soluciones de la ecuación de vorticidad barotrópica en una esfera, la estimación de contaminantes, el control óptimo de emisiones, identificación de intensidad y ubicación de una fuente desconocida.

\section{Introducción}

Las olas en el agua pueden transportar una energía enorme y a menudo son la causa de la erosión de la costa y de la destrucción de los atracaderos y de los barcos en el mar. Las olas gigantes y los tsunamis producen una destrucción especialmente terrible. Pero la energía de las olas también se puede utilizar en beneficio del hombre, si se crea un dispositivo que le permita convertirla en energía eléctrica. Dichos dispositivos permitirían un uso más económico que las fuentes de energía no renovables, como el petróleo, gas y carbón.

El tsunami es uno de los fenómenos naturales más poderosos: una serie de olas marinas muy largas, capaces de cruzar todo el océano a velocidades comparables a la de un avión de pasajeros moderno. Son además catástrofes naturales que pueden causar considerables daños materiales y personales a los seres humanos. La posibilidad de describir este fenómeno y encontrar métodos de previsibilidad de cualquier tipo parece, por tanto, de interés no sólo para los meteorólogos, sino también para los gobiernos, los planes de evacuación o para la industria de seguros, etcétera.

Hasta la fecha, el tsunami más devastador ocurrió el 26 de diciembre de 2004, en el Océano Índico, con un número aproximado de 250,000 víctimas (Kowalik et al., 2005; Levin y Nosov, 2016), el primer lugar de número de afectados entre todos los desastres naturales del siglo xx. Años después, el tsunami que azotó el 11 de marzo 2011 en la costa de Japón provocó 18,453 víctimas humanas (Mori y Takahashi, 2012). 
Un tsunami involucra un grupo de olas de gran energía y de tamaño variable, que se producen cuando se desplaza verticalmente hacia arriba una gran masa de agua por algún fenómeno extraordinario, por ejemplo, un terremoto, erupción volcánica, detonaciones submarinas, deslizamientos de terreno, desprendimientos de hielo glaciar, impacto de meteoritos y otros. Sin embargo, se calcula que $90 \%$ de los tsunamis son provocados por terremotos.

A diferencia de las olas oceánicas normales producidas por el viento o las mareas, que son generadas por la atracción gravitatoria del Sol y la Luna, un tsunami es generado por el desplazamiento de agua. Para que se origine uno, el fondo marino debe ser movido verticalmente de manera abrupta, de modo que una gran masa de agua del océano sea impulsada fuera de su equilibrio normal. Cuando esta masa de agua trata de recuperar su equilibrio genera olas. El tamaño del tsunami estará determinado por la magnitud de la deformación vertical del fondo marino, entre otros parámetros como la profundidad del lecho marino.

Aquí se describen las principales propiedades de la propagación de las olas de tsunami, así como la interacción de las olas con la zona costera. En particular, se explica el mecanismo de compresión de las olas, un aumento en sus amplitudes y una disminución en su velocidad al llegar a la costa. Las conclusiones del análisis cualitativo se ven confirmadas por los resultados analíticos obtenidos al resolver el problema de la llegada de una onda armónica a una costa inclinada. Por último, se discute el colapso de la cresta de la ola en la zona costera. Las conclusiones contienen los principales resultados.

\section{Propiedades de propagación de olas de tsunami}

La vida entera de una ola de tsunami se puede dividir en cuatro etapas sucesivas: 1) iniciación de olas; 2) movimiento a través de la inmensidad del océano; 3) interacción de la ola con la zona costera; 4) rompimiento de la cresta de la ola en la zona costera. Una onda armónica tiene además una serie de parámetros fundamentales (ver figura 1).

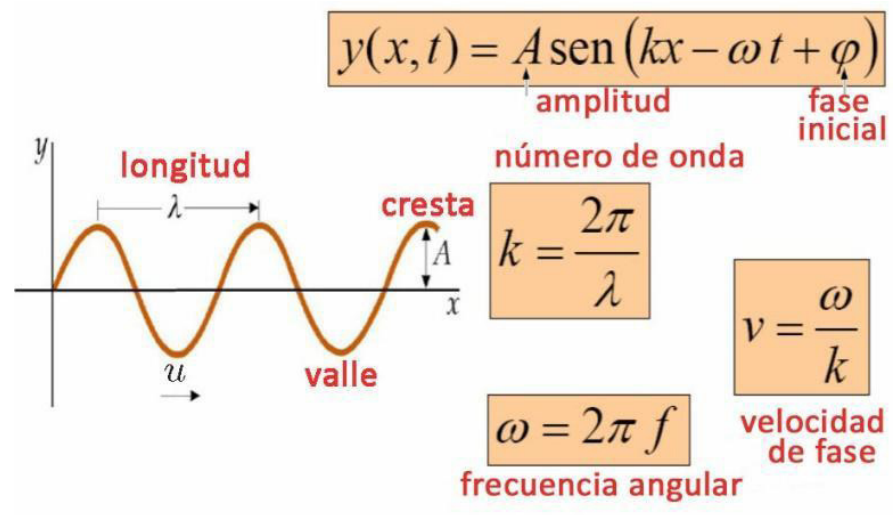


A diferencia de las olas oceánicas ordinarias, que tienen sólo 30 o 40 metros de largo, la longitud de olas de tsunami puede alcanzar cientos de kilómetros. Desde el punto de vista de la hidrodinámica, estas olas se clasifican como ondas de gravedad largas, ya que su longitud excede significativamente la profundidad del océano. Por lo tanto, al estudiarlas, incluso un océano profundo puede tratarse como agua poco profunda, y las ecuaciones de la hidrodinámica se utilizan en la aproximación de "aguas someras" (Skiba, 2009).

Si la longitud de la ola formada es menor que la profundidad del depósito, entonces, sólo la capa superficial participa en el movimiento de la ola. Por el contrario, un tsunami tectónico producido en un fondo oceánico desplazará toda la columna de agua desde el fondo hasta la superficie (ver figura 2). La fricción del agua contra el fondo se vuelve significativa. Las capas inferiores están fuertemente frenadas y no siguen el ritmo de las capas superiores. La velocidad de propagación de olas de tsunami está determinada sólo por la profundidad del agua $\mathrm{H}: \boldsymbol{u}=\sqrt{g H}$ (Pelinovsky, 1996), donde $g=9.8 \mathrm{~m} / \mathrm{s}^{2}$ es la aceleración de la gravedad terrestre. El desplazamiento vertical de la superficie del océano puede ser sólo de unas pocas decenas de centímetros, pero si ocurre en un océano lo suficientemente profundo, la velocidad de las olas será muy alta. Por ejemplo, la velocidad de la propagación de las olas será de $360 \mathrm{~km} / \mathrm{h}$ si la altura es de $10^{3} \mathrm{~m}$, de $720 \mathrm{~km} / \mathrm{h}$ si la altura es de $4 \times 10^{3} \mathrm{~m}$, y de $800 \mathrm{~km} / \mathrm{h}$ si la altura es de $5 \times 10^{3}$. Por lo tanto, la energía transmitida a la ola será enorme.

Figura 2. Formación de un tsunami tectónico.

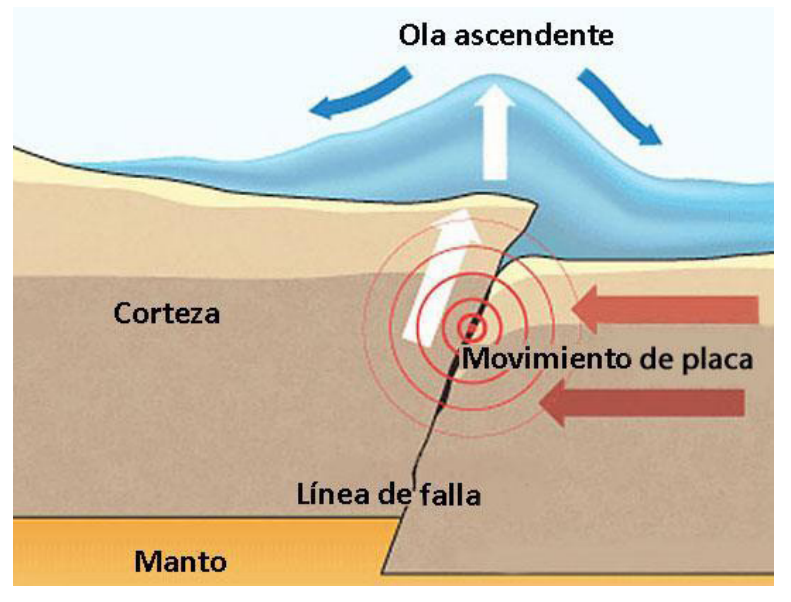

El período de las olas de tsunami (T) varía de varios minutos a varias horas. A partir del período de la onda y la velocidad de su propagación (u), puede calcularse la longitud $\lambda=T u=T \sqrt{g H}$. Una estimación simple basada en esta fórmula muestra que en mar abierto, la longitud de ola del tsunami puede variar de 20 a 2,000 km. Todos los tsunamis se caracterizan por una gran cantidad de energía que transportan, incluso en comparación con las olas más poderosas generadas por el viento. Su amplitud, y por lo tanto su energía, depende de la fuerza de los temblores, de qué tan cerca esté el epicentro del terremoto de la 
superficie del fondo y de la profundidad del océano en un área determinada. En mar abierto, incluso en el caso de eventos catastróficos, la amplitud suele medirse en decenas de centímetros y rara vez supera $1 \mathrm{~m}$. Sin embargo, en la fuente del tsunami, la amplitud del desplazamiento de la superficie del agua puede llegar a 10 m o más, lo que sigue siendo mucho menor que la profundidad del océano. La baja amplitud durante un largo período hace que la ola del tsunami en el océano abierto sea casi invisible para un observador a bordo de un barco o avión, ya que queda camuflada entre las olas superficiales.

Una característica importante que distingue a los tsunamis de otros fenómenos naturales es su capacidad para retener su potencial destructivo cuando se propagan a grandes distancias (hasta 10,000 km o más). Al propagarse en un océano con fondo plano, su amplitud disminuye en $1 / \sqrt{ } r$ (donde $r$ es la distancia del lugar de origen), que es la atenuación mínima posible desde el punto de vista de la ley de conservación de energía. Al mismo tiempo, a diferencia de las olas de tormenta ordinarias que capturan sólo las capas superficiales, en el caso de un tsunami, toda la columna de agua está involucrada en el movimiento desde la superficie hasta el fondo, lo que hace que sea un fenómeno catastrófico grandioso.

\section{Interacción de las olas con la zona costera}

El potencial destructivo de la ola aumenta, alcanzando su máximo cuando llega a la costa. En efecto, cuando un tsunami se acerca a la costa y la profundidad del océano H está disminuyendo, el agua poco profunda comprime la ola, y su longitud (la distancia entre las crestas) se reduce a menos de 20 kilómetros. Al mismo tiempo, la velocidad cae por debajo de los 80 kilómetros por hora porque y debido a la fricción contra el fondo, mientras que la altura de la ola aumenta bruscamente (Yeh et al., 1994).

Describamos brevemente lo que está sucediendo. La imagen aquí es simple: la parte delantera de la ola que emerge en aguas poco profundas desacelera bruscamente, la parte trasera la alcanza y la altura de la ola aumenta. La ola se comprime, su longitud se reduce, y la densidad en la ola se aumenta. Si descuidamos la pérdida de energía debido a la fricción del fondo y la viscosidad del agua, entonces, se conserva la suma de la energía cinética y potencial de la ola. Debido a la disminución de la velocidad de ola, su energía cinética (que es proporcional al cuadrado de la velocidad) disminuye, y parte de ella se convierte en energía potencial. Además, la energía potencial aumenta con la altura de ola (ya que es proporcional a la altura). En consecuencia, estos efectos aumentan la energía potencial. Como resultado, se crea una pared de agua con potencial destructivo, cuya altura puede alcanzar decenas de metros (en el rango de 3 a 30 m). A pesar de que una parte de la energía cinética también se gasta en superar la fuerza de fricción y se convierte en energía interna, la fuerza destructiva del tsunami sigue siendo enorme, lo que, lamentablemente, tenemos que observar periódicamente en varias regiones de la Tierra (ver imagen 1). 


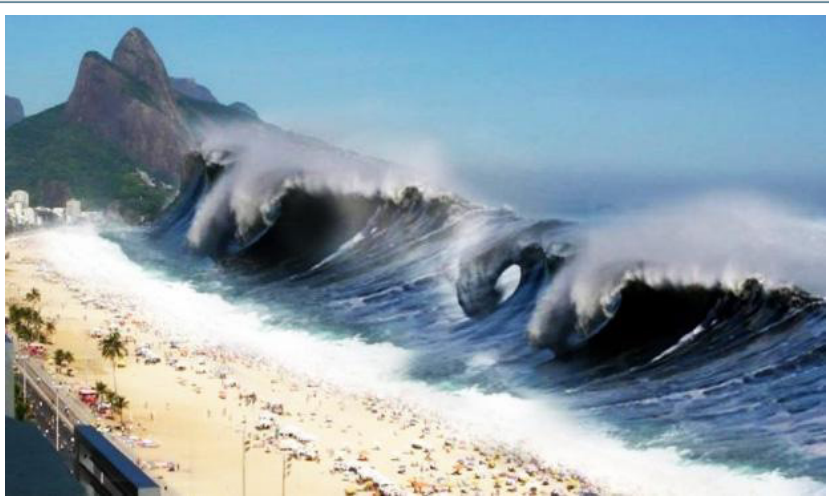

Excepto por los tsunamis muy grandes, la ola que se aproxima no se rompe, sino que parece un maremoto que se mueve rápidamente. Un tsunami fuerte puede incluir varias olas que llegan en unas pocas horas, con un intervalo de tiempo significativo entre las crestas de las olas. Además, la primera ola a menudo no es la más fuerte. El descuido o ignorancia de esta propiedad del tsunami a menudo conduce a la pérdida de vidas.

\section{Salida de ola a costa inclinada: solución exacta}

Supongamos que el fondo marino tiene la forma (ver la figura 3):

$$
h_{T}(x)=\left\{\begin{array}{ccl}
(H / L) x & \text { si } & 0 \leq x \leq L \\
H & \text { si } & x \geq L
\end{array}\right.
$$

Consideremos una ola larga que tiene amplitud pequeña a y se acerca la costa desde mar abierto, teniendo en el punto extremo $x=L$ la forma de onda armónica:

$$
\xi(L, t)=A \cdot \cos (\sigma t+\phi)
$$

Supongamos que el fondo marino es moderadamente inclinado: $H / L=\tan \beta \approx \beta$, es decir el ángulo $\beta$ es pequeño. Usando un modelo hidrodinámico de "aguas someras", Billingham y King (2000) encontraron la solución exacta (analítica) de este problema en términos de las funciones de Bessel (Zwillinger, 2003) (ver figura 4). A medida que la ola se acerca a la costa en $x=0$, su amplitud crece y su longitud disminuye. Sin embargo, se debe notar que la aproximación de "aguas someras" no funciona cuando $x$ es bastante pequeño y las olas se están rompiendo (ver la siguiente sección).

Figura 3. Una ola que llega a la

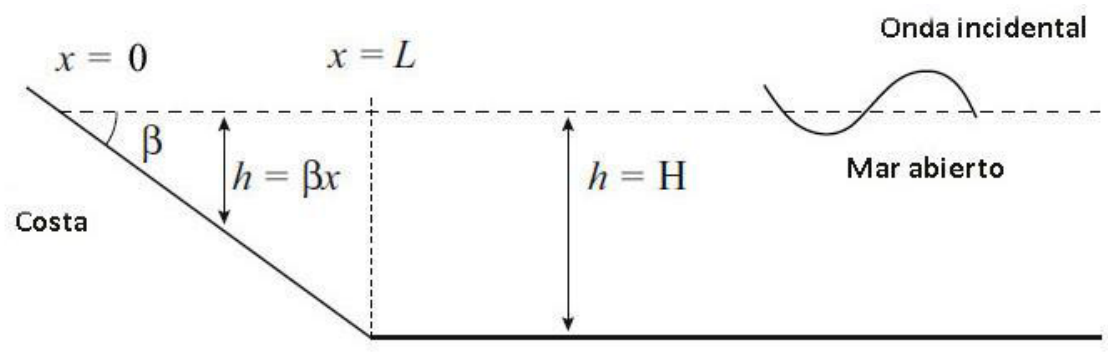


Figura 4. Amplificación de la amplitud de onda y disminución de su longitud.

Figura 5. Evolución del perfil de la ola del mar rompiendo.

Figura 6. Colapso de ola de tsunami al entrar en aguas poco profundas.

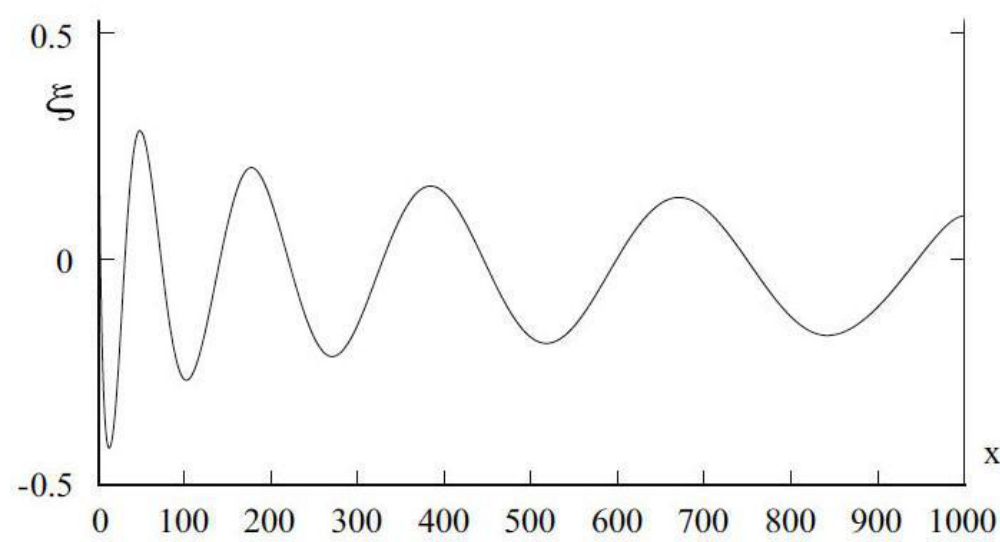

\section{Colapso de la cresta de la ola en la zona costera}

A diferencia de las olas de viento, cuando sólo vibra una capa de agua superficial, en el caso de las olas de tsunami, el agua se mueve hacia adelante y hacia atrás a lo largo de todo el espesor del océano, hasta el fondo. Es por eso que la topografía del fondo del océano es tan importante para su propagación. Tan pronto como la ola entra en aguas poco profundas ( $H$ disminuye de manera drástica), la velocidad desciende bruscamente: a una profundidad de 10 m, la velocidad es de sólo $10 \mathrm{~m} / \mathrm{s}$. Así, uno de los principales efectos de la llegada de una ola a costa es un aumento de su altura.

Ahora consideraremos el

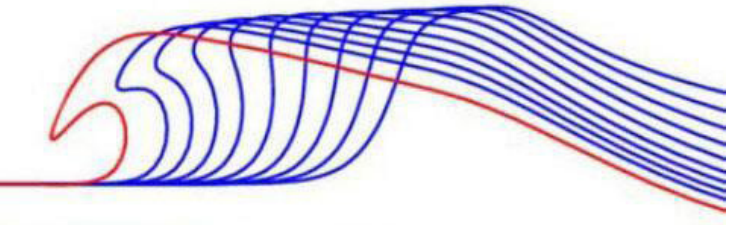
segundo efecto: el rompimiento de la ola (ver figura 5). Éste es un aspecto no lineal de las olas en aguas poco profundas.

En una forma simplificada, la imagen se ve así. Aquí la altura a entre la cresta y el valle de la ola debe entenderse como la profundidad local (ver figura 6). Esto significa que, en comparación con el valle (donde la velocidad es $u=\sqrt{g H}$ ), la cresta tiene una velocidad más alta ( $u=\sqrt{g(H+a)})$ e intenta alcanzar el valle. En el momento en que la cresta alcanza el frente, se produce un colapso. Está claro que cuanto menos es la profundidad $H$, más fuerte es este efecto (ya que aumenta $a$ al disminuir $H$ ).

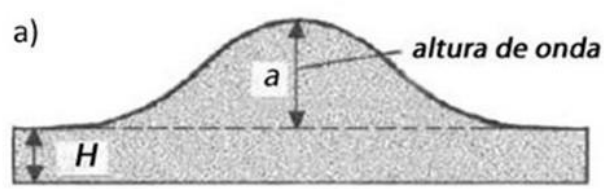

b)

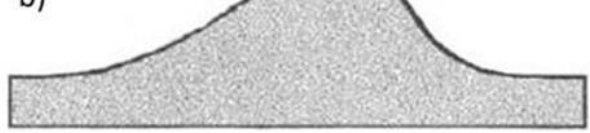

c)

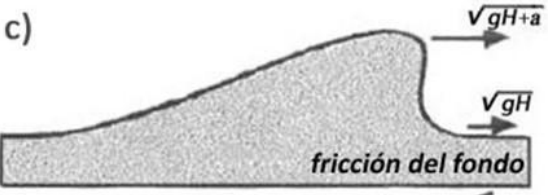

d) Ola rompiendo flujo inverso 
Figura 7. Zonas de la ola del mar rompiendo.

Imagen 2. Rompimiento de la ola en la costa.
Además, la velocidad de la parte inferior de la ola se frena por la fricción del fondo del mar y el flujo inverso que aparece después del rompimiento de la ola anterior (ver figura 7). Por lo tanto, al acercarse a la costa, la parte superior de la ola no sólo se eleva, sino que también tiende a volcarse hacia adelante (ver imagen 2).

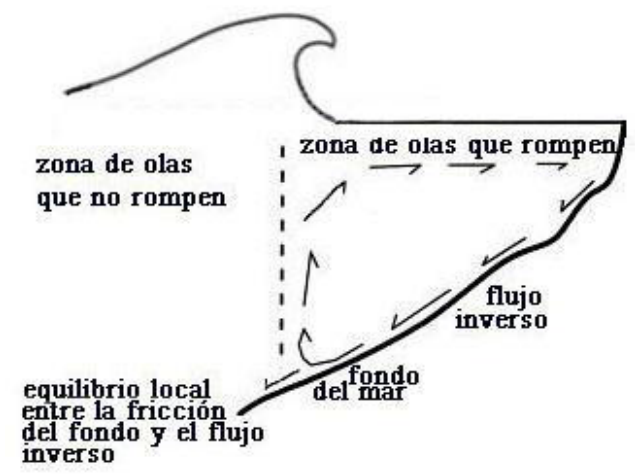

La mecánica de fluidos dicta que la estructura de las olas rompientes depende principalmente de la pendiente de la batimetría de la costa. Existen diferentes clasificaciones de olas rompientes (Carrier et al., 2003; Madsen y Schäffer, 2010).

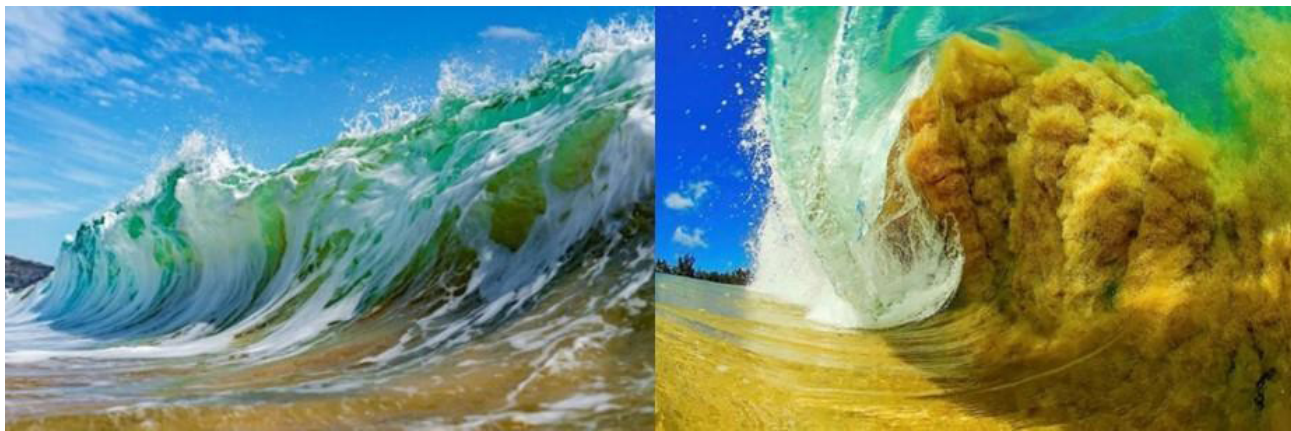

\section{Conclusiones}

En este artículo se vio que cuando un tsunami entra en aguas poco profundas cerca de la costa, la velocidad de las olas desciende bruscamente con la profundidad, su longitud disminuye (las olas se comprimen) y su amplitud aumenta. Como resultado, la energía potencial de las olas aumenta (ya que es proporcional a la altura de las olas), además, parte de la energía cinética de las olas (que es proporcional al cuadrado de su velocidad) también se convierte en su energía potencial y, por tanto, la fuerza destructiva de las olas aumenta. También se estudió un proceso no lineal que es característico de muchas olas del mar y del océano, a saber, el rompimiento de las crestas de las olas cuando llegan a la costa.

La energía de las olas del océano se puede utilizar para realizar trabajos útiles: generar electricidad, desalar agua y bombear agua a los tanques. La energía de las olas es una fuente inagotable de energía. Tener en cuenta este efecto también es importante en el diseño de estructuras de protección costera. 


\section{Referencias}

Billingham, J. y King, A. C. (2000). Wave Motion. Cambridge University Press.

* Carrier, G. F., Wu, T. T. y Yeh, H. (2003). Tsunami run-up and draw-down on a plane beach. J. Fluid Mech., 475, 79-99. https://doi.org/10.1017/S0022112002002653

Kowalik, Z., Knight, W., Logan, T. y Whitmore, P. (2005). Numerical Modelling of the Global Tsunami: Indonesian Tsunami of 26 December 2004. Science of Tsunami Hazard, 23(1), 40-56. https://cutt.ly/MWPPJA7

- Levin, B. y Nosov, M. (2016). Physics of Tsunamis. Springer Intern. Publ.

* Madsen, P. A. y Schäffer, H. A. (2010). Analytical solutions for tsunami runup on a plane beach: single waves, N-waves and transient waves. J. Fluid Mech, 645, 27-57. https://doi.org/10.1017/S0022112009992485

- Marchuk, A. G. (2020). The Tsunami Wave Energy. Vestnik NSU. Information Technologies, 18(4), 39-53. https://doi.org/10.25205/1818-7900-2020-18-4-39-53

* Mori, N. y Takahashi, T. (2012). The 2011 Tohoku Earthquake Tsunami Joint Survey Group. Nationalwide post event survey and analysis of the 2011 Tohoku Earthquake Tsunami. Coast. Eng. J., 54 (1), pp. 1250001-1-1250001-27. https://doi. org/10.1142/S0578563412500015

* Pelinovsky, E.N. (1996). Hydrodynamics of tsunami waves. Waves in Geophysical Fluids. Eds. J. Grue, K. Trulsen, Springer, pp. 1-48. https://doi.org/10.1007/978-3211-69356-8_1

* Skiba, Y. N. (2009). Introducción a la Dinámica de Fluidos. Dirección General de Publicaciones y Fomento Editorial, unAm.

* Yeh, H., Liu, Ph., Briggs, M. y Synolakis, C. (1994). Propagation and Amplification of Tsunamis at Coastal Boundaries. Nature, 372, 353-355. https://doi. org/10.1038/372353a0

* Zwillinger, D. (Ed.). (2003). Standard Mathematical Tables and Formulae. Chapman \& Hall; CRC Press.

\section{Cómo CITAR ESTE ARTículo}

* Skiba, Y. N. (2021, septiembre-octubre). Dinámica de olas del tsunami. Revista Digital Universitaria (RDU), 22(5). http://doi.org/10.22201/cuaieed.16076079e.2021.22.5.6 